\title{
Diagnosis of deep venous thrombosis and pulmonary embolism
}

\author{
NICHOLAS S. MACKLON MBChB, MRCOG, MD
}

Senior Lecturer

Department of Obstetrics and Gynaecology, University Hospital Rotterdam, The Netherlands

\begin{abstract}
Accurate diagnosis of deep venous thrombosis (DVT) and pulmonary embolism (PE) is required because treatment can be life-saving, while inappropriate anticoagulation exposes the mother and fetus to haemorrhage and other hazards. Clinicians must be aware of which patients are at risk because DVT is frequently asymptomatic. Clinical diagnosis is unreliable for DVT and PE so objective tests are required. Venography is the gold standard test for DVT but is invasive and has been superseded by less invasive tests such as duplex ultrasound which is now the first-line investigation in pregnancy. However, where doubt remains, venography, CT and MRI have a role. Ventilation-perfusion scanning is the pivotal test for PE in pregnancy, and need not expose the fetus to excess radiation. If the result is unclear deep venous ultrasound can guide management of suspected PE, thus avoiding pulmonary angiography, the invasive gold standard test.
\end{abstract}

Key words: diagnosis; venous thrombosis; pulmonary embolism; pregnancy.

Pulmonary thrombo-embolism (PTE) remains a major cause of maternal mortality (DHSS, 1996). Accurate diagnosis is vital as untreated pregnant patients with deep venous thrombosis (DVT) have a mortality rate of at least $15 \%$. With early accurate diagnosis and anticoagulant therapy, this mortality is reduced to $0.7 \%$ (Villasanta, 1965). Conversely, a false-positive diagnosis of PTE and inappropriate anticoagulation exposes the patient to other severe hazards such as haermorrhage, osteoporiosis and heparininduced thrombocytopenia (Ginsberg et al, 1989b). In recent years, new techniques for the diagnosis of DVT and PE which offer obstetricians the means of objective diagnosis at minimal or no risk to the fetus have become available. In this chapter, these methods will be reviewed.

\section{DEEP VENOUS THROMBOSIS}

\section{Clinical assessment}

The sensitivity and specificity of clinical diagnosis of DVT is known to be poor. Fewer than $50 \%$ of cases of DVT involving major proximal veins are 
recognizable clinically, while venography substantiates the diagnosis in only about $40 \%$ of patients with clinical findings compatible with DVT (Genton and Turpie, 1980; Ramsey, 1983). The role of clinical assessment lies principally in the recognition of the patient at risk.

A good medical history and careful physical examination can identify risk factors additional to those imposed by pregnancy which should increase the suspicion of DVT. Age over 35 is associated with a doubling in incidence, and emergency caesarean section is the mode of delivery associated with the highest incidence of post-natal DVT (Macklon and Greer, 1996). The effect of risk factors is cumulative (Wheeler et al, 1982) and clinicians need to be alert to the likelihood of DVT in women with multiple risk factors because many women with DVT are asymptomatic.

Many symptoms and signs have been described as representing manifestations of DVT. While most occur as a result of venous outflow obstruction and inflammation of the venous walls and surrounding tissues, none is unique to DVT and all can be caused by non-thrombotic conditions (Fairbarn, 1963). Unilateral leg swelling considered one of the most reliable physical findings in non-pregnant patients (Wheeler and Anderson, 1995 ) is of less value in pregnancy due to its frequent physiological occurrence.

While careful clinical examination may enable identification of other obvious disorders which may mimic DVT-such as cellulitis or sciatic pain - the decision to initiate or withhold anticoagulation should be based on objective testing.

\section{Venography}

Although ascending venography remains the 'gold standard' for the diagnosis of DVT, it has a number of limitations. Some $10-30 \%$ of venograms fail to visualize some segment of the venous system. Because most DVT occurring in pregnancy form in the ileofemoral segment (Bergqvist and Hedner, 1983; Polak and Wilkinson, 1991), non-visualization of the iliac system arising from dilution of the contrast agent is of particular importance. Further, considerable inter-observer variation in interpretation has been demonstrated (Couson et al, 1993; Illescas et al, 1993) and at least $10 \%$ of contrast venograms performed in non-pregnant subjects are inadequate for interpretation (Huisman et al, 1986). The risks associated with venography include pain at the time of injection of contrast, hypersensitivity to the medium, and extravasation of the medium resulting in damage to the skin of the foot. Thrombosis can occur secondary to venography owing to the irritant effects of the hyperosmolar media on the venous endothelium. Lowering the osmolality of contrast media can reduce the irritant effects and subsequent DVT formation (Bettman and Paulin, 1977).

For obstetricians, however, concerns are principally over the safety of the fetus. Pelvic and abdominal shielding with lead aprons can protect the fetus from the hazards of radiation exposure, but visualization of the iliac veins may be compromised. Ginsberg et al (1989a) have demonstrated that 
shielded unilateral venography results in $<0.05 \mathrm{rad}$ being absorbed by the fetus as opposed to $0.314 \mathrm{rad}$ when no shielding is provided. The literature on fetal risks from in-utero exposure to radiation suggests a slight increase in the risk of childhood cancer following in-utero exposure to radiation doses of less than $5.0 \mathrm{rad}$, but no increase in congenital malformations or increase in stillbirths and infant deaths appears to follow such radiation exposure (Ginsberg et al, 1989a). While clinicians continue to avoid venography in pregnancy, the test can be of value and the risks should be kept in perspective.

\section{Impedance plethysmography (IPG)}

IPG is a safe, inexpensive and non-invasive test that measures changes in electrical impedance resulting from changes in blood volume within a limb. Following the inflation of a thigh cuff, the venous outflow is occluded. In the absence of any obstruction to venous flow, release of the cuff results in a rapid outflow of blood resulting in an increase in resistance as measured between two electrodes placed around the limb. In the presence of venous thrombosis the rate of emptying is reduced. In non-pregnant patients IPG has been shown to have a sensitivity ranging from 63 to $95 \%$ and a specificity of 83 to $96 \%$ in the detection of proximal occlusive venous thrombi (Hull et al, 1984; Anderson et al, 1993), and withholding anticoagulation from non-pregnant patients with serially negative IPG test has been shown to be safe (Hull et al, 1985; Huisman et al, 1986). The value of IPG in pregnancy is less clear. False-positive results arising from obstruction to venous return by the gravid uterus can occur, although these can be prevented by examining the patient in the left lateral position (Ginsberg et al, 1988). However concern over the poor sensitivity in the diagnosis of non-occlusive proximal thrombi and calf thrombi (Hull et al, 1976) and the development of other non-invasive tests have diminished the role of IPG in pregnancy.

\section{Duplex Doppler-ultrasound}

Ultrasound offers a quick non-invasive means of assessing venous thrombo-embolic disease and is now the first choice investigation for DVT in pregnancy. Thrombus itself can be visualized in the occluded segment of the vessel (Aitken and Godden, 1987), but in practice such a finding is difficult to interpret. Clot echogenicity is variable (Murphy and Cronan, 1990), and slow-flowing blood can appear sufficiently echogenic to mimic the appearance of the clot (Machi et al, 1983). Although acute thrombus may be echoluscent, it frequently gives rise to local distension of the vessel. The presence of intraluminal thrombus can be confirmed by gently compressing the vessel with the ultrasound transducer. If no thrombus is present then coaptation of the vein walls will be seen.

The compression technique is the principal means of ultrasound assessment for DVT. The deep veins may be examined from the level of the inguinal ligament to the bifurcation of the popliteal vein (Aitken and 
Godden, 1987). The femoral veins are best evaluated in the supine position while the popliteal veins may be examined in the decubitus position or in the semi-supine position with the legs externally rotated and slightly flexed at the knee. The gain should be set so that normal vessels appear free of internal echoes. Compression is best applied in the transverse axis as the transducer will not roll off the vessel. The pressure applied should be sufficient only to dimple the overlying skin. If the artery is compressed, excessive pressure has been used. The lumen of a thrombus-free vein completely collapses on compression (Figure 1), while DVT prevents coaptation of the venous wall (Cronan et al, 1987). In the symptomatic patient, clot usually extends over one or more venous segments (Markel et

(a)

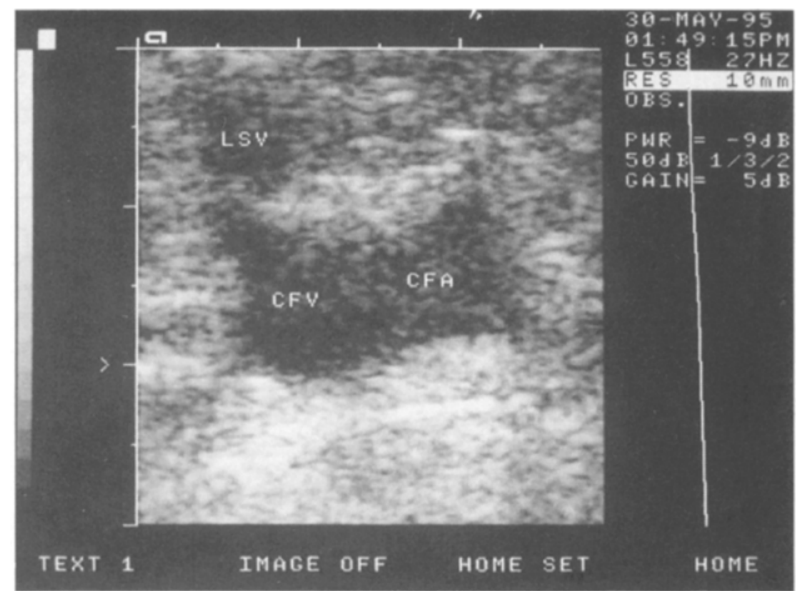

(b)

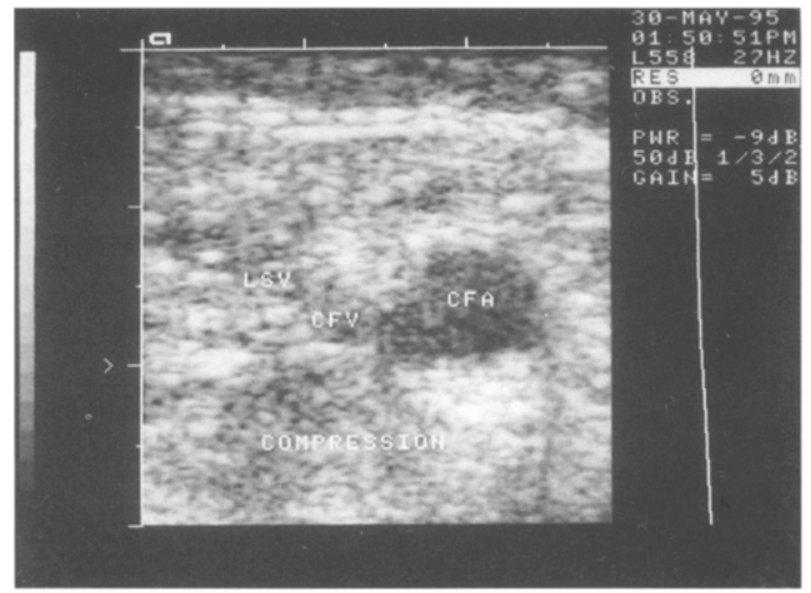

Figure 1. The lumen of the common femoral vein (CFV), imaged in transverse section (a), has collapsed on application of compression, indicating a thrombus-free segment (b). 
al, 1992) so that normal compressibility at $2 \mathrm{~cm}$ intervals may be interpreted as a thrombus-free vessel (Cronan, 1993).

Compression ultrasound has been extensively validated against venography and found to have a very high positive predictive value for DVT (Cronan et al, 1987; Lensing et al, 1989). The pooled sensitivity from many studies in non-pregnant patients shows compression ultrasound to have a sensitivity of $95 \%$ for detecting lower extremity DVT and a specificity of 98\% (Cronan, 1993). Studies assessing the outcome after a negative compression ultrasound scan have also demonstrated the reliability of the technique (Sarpa et al, 1989; Vaccaro et al, 1990). More recently, a large prospective randomized trial comparing serial compression ultrasonography with serial impedance plethysmography for the diagnosis of DVT using contrast venography as a reference has confirmed the positive predictive value of an abnormal ultrasound test to be $94 \%$ compared to $83 \%$ for impedance plethysmography. In non-pregnant patients with repeatedly normal results, the incidence of venous thrombo-embolism during the 6-month follow-up period was $1.5 \%$ for compression ultrasound and $2.5 \%$ for serial impedance plethysmography (Heijboer et al, 1993).

The limitations of compression ultrasound in pregnancy relate principally to the difficulty in assessing the ileo-femoral veins as they pass behind the gravid uterus. Further, in the area of the inguinal ligament and in the adductor canal, complete compression of the vessel may be difficult without applying additional, and possibly painful, compressive force. Indirect methods of assessing proximal venous patency are therefore required.

The Valsalva manoeuvre normally causes a $50-200 \%$ increase in the diameter of the common femoral vein. If thrombus is present the response is absent or limited, so an increase of less than $10 \%$ may indicate femoral venous obstruction (Effeney et al, 1983). However, the response rapidly diminishes in the more distal parts of the leg. Excellent patient co-operation is required for adequate performance of this technique, and although a normal increase in vein diameter excludes occlusive DVT, an abnormal response is not specific for thrombus. The gravid uterus does not appear to prevent the Valsalva response at the femoral vein and it has been described as a diagnostic method in pregnancy (Duddy and McHugo, 1991). However, in practice it has been found to be of limited reliability (Comerota et al, 1993)

A more effective means of assessing proximal patency is to observe whether venous flow velocity at the common femoral vein fluctuates with respiration. This can easily be visualized using duplex Doppler ultrasound (Figure 2) and indicates a patent iliac vein (Killewich et al, 1988). The absence of flow indicates proximal occlusion (Figure 3). Occlusion of the vessel by thrombus lying distal to the segment being assessed can be detected by gently squeezing the calf. This transiently augments the velocity of venous flow and is detected as a rapid and transient increase in the Doppler shift as the accelerated blood passes through the insonated segment of vessel and appears to be a safe and reliable means of assessing the distal vessels of the leg for occlusive thrombus (Cronan, 1993). 


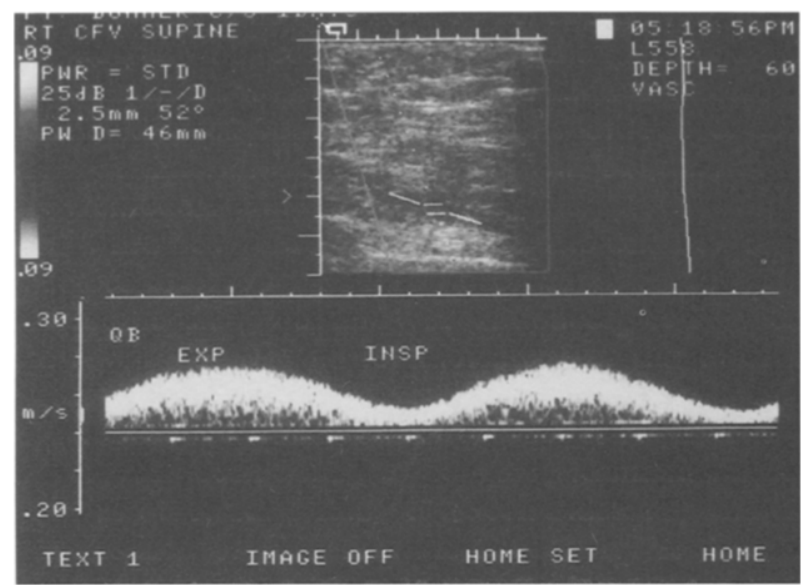

Figure 2. Fluctuation in blood flow velocity with respiration indicates proximal venous patency.

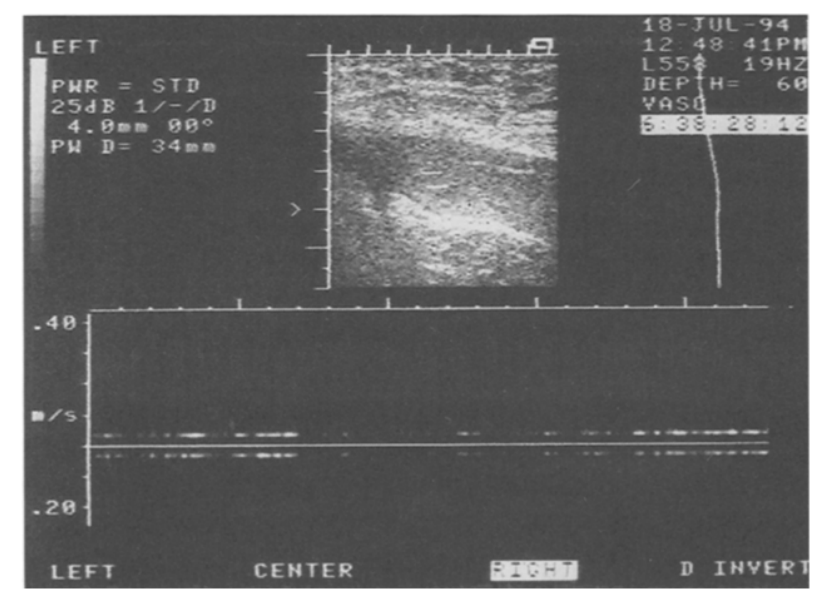

Figure 3. In the presence of proximal occlusive thrombus, no flow is observed through the sampled venous segment.

Duplex ultrasound is now being successfully employed for the diagnosis of DVT in pregnancy (Greer et al, 1990; Polak and Wilkinson, 1991), and a recent longitudinal study has aided interpretation by describing the normal ultrasound appearance of the deep veins in pregnancy (Macklon et al, 1997). The diameter of the normal CFV increases from a mean of $9.1 \mathrm{~mm}$ to $12.7 \mathrm{~mm}$ by term, while the flow velocity falls from a mean of $14.8 \mathrm{~cm} / \mathrm{s}$ in early pregnancy to $4.5 \mathrm{~cm} / \mathrm{s}$ by the mid-third trimester. There appears to be little effect of increasing gestation on the amplitude of respiratory flow fluctuation, making this indirect means of assessing the proximal iliac system valuable throughout pregnancy. Adoption of the lateral position leads 
to a marked reduction in the diameter of the opposite CFV, allowing discrimination of the physiological distension of pregnancy from pathological distension secondary to DVT. This manoeuvre has also been shown to increase the amplitude of respiratory flow fluctuation and may be a useful adjunct when iliac thrombus is suspected (Macklon et al, 1997).

Colour Doppler ultrasound offers a potential enhancement to the diagnostic power of ultrasound in pregnant patients. Venous flow velocities can be detected on modern ultrasound machines by colour Doppler mapping. In the absence of thrombus, the vessel lumen should be seen to fill with colour during spontaneous flow, while the presence of local or proximal thrombus will alter the colour signal. If doubt persists, distal augmentation of flow velocity by means of calf compression may result in clear filling of the vessel lumen with colour. If the lumen remains free of a colour shift signal despite augmentation, then thrombus is likely to be present. Partial filling may indicate the presence of local non-occlusive thrombus. Colour Doppler has been shown to have a sensitivity and specificity similar to that of duplex and compression ultrasound. The benefits appear to reside principally in the reduction in time required to assess the veins, and in the ability to identify and examine smaller veins for thrombus such as calf veins (Rose et al, 1990; Mattos et al, 1992).

There are no prospective studies available which have evaluated the sensitivity and specificity of ultrasound in detecting DVT in pregnancy neither are there any published data assessing the safety of withholding anticoagulants in pregnant patients with serial negative ultrasonography. However, the data available indicate that ultrasound assessment can be used as the initial test in pregnant women with suspected DVT.

\section{Magnetic resonance imaging}

In recent years, the use of magnetic resonance imaging (MRI) in the detection of centrally located DVT has been described. In comparisons against venography it has been shown to have a sensitivity of $97-100 \%$ and a specificity of $95 \%$ in non-pregnant subjects (Evans et al, 1993; Spritzer et al, 1990, 1993). It appears to offer superior visualization of the proximal extent of thrombus and, unlike venography, can distinguish acute from chronic thrombus (Erdman et al, 1990). Spritzer et al (1995) have recently reported its use in pregnancy. Although it is non-invasive and does not expose the fetus to radiation, safety for the fetus has not yet been confirmed (Kanal et al, 1990) and its role in pregnancy remains to be established.

\section{Light reflection rheography (LRR)}

LRR is a refined form of photoplethysmography which uses infrared light of $940 \mathrm{~nm}$ wavelength, emitted from diodes in the probe, and a sensor which measures light reflected from the skin dermal microcirculation. The intensity of the reflected light establishes a graphic pattern that indirectly quantifies parameters of venous function by measuring changes in the microcirculation. LRR is inexpensive, does not require operator calibration 
and is not operator-dependent. It has been shown to be as accurate as other non-invasive tests in the diagnosis of deep venous thrombosis in nonpregnant patients (Thomas et al, 1991) and would therefore appear to offer considerable promise as a non-invasive diagnostic and screening tool for clinically suspected DVT in pregnancy. Studies in non-pregnant patients have indicated a sensitivity of up to $96 \%$ and a specificity of $83 \%$ when compared to venography. A recent study indicated the utility of the technique in pregnancy (Allbert et al, 1991). However, the effects of gestational changes on the interpretation of LRR have yet to be fully elucidated and further assessment of this technique in pregnancy and the puerperium is required.

\section{Calf vein thrombosis}

The clinical importance of calf vein thrombi remains controversial. While there is now a considerable body of evidence suggesting that significant emboli do not arise from isolated calf veins (Cohen et al, 1988; Meibers et al, 1988; Philbrick and Becker, 1988), proximal propagation has been demonstrated in duplex ultrasound follow-up studies (Lohr et al, 1995). Venogram studies in non-pregnant patients have indicated that $40 \%$ of calf clots remain isolated, $40 \%$ quickly lyse and $20 \%$ extend upward. Cogo et al (1993) found isolated calf-vein DVT in only $12 \%$ of 562 consecutive venograms, while occlusive proximal venous thrombi were present in $88 \%$ of those with symptomatic DVT. Their data and those from other studies comparing limited compression ultrasound (confined to the common femoral and popliteal veins) to full-leg ultrasound examination suggest that, in non-pregnant subjects, examination of the proximal leg veins will detect most symptomatic DVT (Pezzulo et al, 1996).

Given the continuing uncertainty over the clinical importance of isolated calf-vein thrombosis, and the possible relationship between calf-vein thrombi and post-phlebitic syndrome (Lagerstedt et al, 1985; Lohr et al, 1991), those women who have continuing or worsening calf symptoms after a negative proximal vein scan should be offered a follow-up scan at 2 days in order to detect any extension of thrombosis into the popliteal segment.

\section{Ovarian vein thrombosis}

The diagnosis of this rare condition which affects approximately 1 in 2000 pregnancies (Dunnihoo et al, 1991) is complicated by the broad differential diagnosis which essentially includes all causes of acute lower abdominal pain. Most cases of ovarian vein thrombosis (OVT) occur in the first 7 days post-partum (Munsick and Gillanders, 1981) and the diagnosis should be considered in any woman re-admitted after an uncomplicated vaginal delivery presenting with a picture suggestive of endometritis or pyleonephritis. Classically, a combination of fever and lower abdominal pain is present, together with a tender lower sausage-like or rope-like abdominal mass (Witlin and Sibai, 1995). Imaging is usually required to confirm the diagnosis. The presence of overlying bowel gas may make ultrasound 
imaging difficult, and CT or MRI scanning is normally required (Simons et al, 1993). The latter is clearly preferable in antenatal pregnant patients with suspected OVT because it avoids exposure of the fetus to ionizing radiation. In practice, however, the diagnosis is frequently made at laparotomy for suspected appendicitis (Simons et al, 1993).

\section{PULMONARY EMBOLISM}

The problems relating to the diagnosis of pulmonary embolism in pregnancy are essentially those of DVT. Clinical diagnosis is unreliable. Pregnancy is less likely to alter the clinical presentation of PE than of DVT, but the symptoms and signs are, as in the non-pregnant patient, nonspecific. Dyspnoea is the most common symptom but pleuritic chest pain may also occur in the presence of pleural inflammation due to infarction and can be associated with haemoptysis. Massive pulmonary thromboembolism may cause oppressive chest pain and can lead to cardiovascular collapse, syncope, acute cor pulmonale and loss of consciousness.

Clinical examination is non-specific. A rapid respiratory rate is usually present but only a small proportion of patients will demonstrate a friction rub. Massive embolism may be associated with cyanosis, hypotension and elevated jugular venous pressure but less common and less dramatic manifestations such as wheezing or unexplained fever should also raise clinical suspicion. Given the frequently vague clinical presentation of PE and the potentially life-saving effects of early heparin therapy balanced against the hazards of inappropriate anticoagulation in pregnancy, early recourse to objective diagnostic testing is mandatory.

\section{Initial investigations}

Chest radiography has a low sensitivity and specificity for PE (Nymen, 1993) but can differentiate conditions such as pneumothorax. A recent chest $\mathrm{X}$-ray is required when interpreting lung scans in order to differentiate true perfusion defects from local pulmonary infiltrates, pleural effusions or cardiomegaly. The role of electrocardiography is to exclude myocardial infarction as it is an insensitive and non-specific means of diagnosing PE (Szucs et al, 1971). Blood gas estimation is of limited value as a means of diagnosing $\mathrm{PE}$ because a normal $\mathrm{PO}_{2}$ may be found in up to $15 \%$ of cases of PE (Bell et al, 1977) and a low $\mathrm{PO}_{2}$ may indicate the presence other pathology such as amniotic fluid embolism in the post-partum woman. The risk of haemorrhage from an arterial puncture site in the presence of anticoagulation is substantial, and pulse oximetry offers a less invasive means of measuring arterial oxygenation.

\section{Ventilation-perfusion lung scintigraphy}

The ventilation-perfusion scan (VQ scan) is the pivotal test in the diagnostic work-up of suspected PE. The perfusion scan is normally performed 
first because a normal result, while not completely excluding PE, means that the patient can be safely left untreated because any emboli present are unlikely to be clinically significant, and the further radiation dose associated with the ventilation scan can be avoided (Juni and Alavi 1991). The ventilation component should not, however, be omitted if perfusion defects are identified because defects in lung perfusion may arise in the presence of conditions other than $\mathrm{PE}$, such as pneumonia and chronic obstructive airways disease. Classical PE produces a perfusion defect with no corresponding ventilation defect or CXR abnormality: a segmental 'mismatch'. Unfortunately this pattern may be observed in other lung diseases, including pneumonia, carcinoma, amniotic fluid embolism and mitral valve disease (Velchik et al, 1989). Results are therefore interpreted and expressed in terms of the probability of PE being present: high, intermediate, low or normal.

The radiation exposure to the fetus can be minimized in the first instance by withholding ventilation studies if the perfusion scan is normal. Perfusion scans employ technetium macro-aggregates of human albumin $\left({ }^{99 \mathrm{~m}} \mathrm{Tc}-\right.$ MAA) or microspheres which remain in the lung or are transported to the liver or spleen via the reticulo-endothelial system from where radiation can be absorbed by the fetus. The dose of ${ }^{99} \mathrm{~m}$ Tc-MAA normally employed is 3 millicuries $(\mathrm{mCi})$ but this can be reduced by using 1.0 or $2.0 \mathrm{mCi}$ without compromising the quality of the scan (Ginsberg et al, 1989a). A combined ventilation perfusion scan need subject the fetus to no more than 0.01 rads, a dose considered to pose a negligible risk of oncogenicity or teratogenicity to the fetus (Ginsberg et al, 1989a).

\section{Pulmonary angiography}

Pulmonary angiography is considered to be the most accurate means of diagnosing PE (Juni and Alavi, 1991) but is invasive and usually avoided in pregnancy. The diagnosis of $\mathrm{PE}$ is made by visualizing the embolus as a constant intraluminal filling defect or by finding an abrupt cut-off of a pulmonary artery greater than $2.5 \mathrm{~mm}$ in diameter (Kearon and Hirsh, 1995). Studies relating to risks for the patient are based on non-pregnant subjects but show an incidence of serious complications of $1.5-3 \%$, and a mortality of $0.2 \%$ (Mills et al, 1980; Perlmutt et al, 1987). With modern techniques and the use of low-osmolality contrast media these risks may be reduced. Minor side-effects include flushing and transient hypotension. Pulmonary angiography is also limited by its technical complexity and costs, and it is not available in many hospitals.

\section{The role of venous studies}

On the basis of the presence of underlying risk factors, symptoms, clinical findings and the results of initial investigations, a clinical probability of $\mathrm{PE}$ can be estimated. Including a clinical assessment of prior probability of PE may reduce the need for pulmonary angiography in cases where the result of the VQ scan is inconclusive. However, such judgements are complex 
and, by their nature, subjective. The probability of PE in those with a highprobability VQ scan is 85\% (PIOPED, 1990) and is high enough to merit anticoagulant therapy without recourse to further investigations. Conversely a normal or near-normal VQ scan is associated with pulmonary embolism in 4\%. Unless clinical suspicion is high, it would appear reasonable to withhold anticoagulation from this group of patients without further testing. If the VQ scan is non-diagnostic (intermediate or low probability) duplex ultrasound scanning of the proximal deep veins of the leg should be performed to determine whether or not DVT is present. If not, then treatment can be withheld without recourse to pulmonary angiography. A recent study has suggested that the use of serial leg scans on those with nondiagnostic VQ studies can remove the need for pulmonary angiography in up to $60 \%$ of such patients (Stein et al, 1995). Although some patients with PE do not show DVT with single or serial leg scans, studies in nonpregnant subjects have shown that only $0.6 \%$ of such patients will suffer recurrent $\mathrm{PE}$ while only $0.2 \%$ will suffer fatal recurrent $\mathrm{PE}$ (Hull et al, 1994). Given the risks of pulmonary angiography and inappropriate anticoagulation, such a strategy would appear to be of particular value in pregnant patients with suspected PE. Clinical validation of this strategy in pregnant subjects has not been carried out, however, and if doubt persists in cases with non-diagnostic VQ scans, pulmonary angiography should be considered.

\section{Alternative techniques}

The measurement of plasma D-Dimer offers a biochemical means of detecting PE. D-Dimer is a degradation product formed when cross-linked fibrin is lysed by plasmin. Two recent studies in non-pregnant subjects indicate that cut-off levels of $<500 \mathrm{ng} / \mathrm{ml}$ (Bounameaux et al, 1991) and $<300 \mathrm{ng} / \mathrm{ml}$ (Demers et al, 1992) had a negative predictive value of $98 \%$ for PE. A low level of D-Dimer might therefore safely exclude PE. However, studies are still required to assess the safety of withholding treatment in patients with low D-Dimer levels. Interpretation in pregnancy is complicated by the effect of pregnancy on background levels of D-Dimer which appear to increase slightly with increasing gestational age (Francalanci et al, 1995).

Studies in non-pregnant subjects have indicated that CT scanning may offer an alternative to pulmonary angiography when doubt persists over the presence of PE after full diagnostic work up (Teigen et al, 1993). However, it has not been assessed in pregnancy.

\section{CONCLUSION}

Recognition of the at risk patient, combined with early use of objective testing and the institution of effective therapy are required if any impact on the mortality and long-term morbidity associated with thrombo-embolic disease is to be achieved. In recent years, non-invasive and accurate 
objective tests for DVT and PE have become widely available. While they have been extensively validated in non-pregnant subjects, such validation is unlikely to be carried out in pregnant patients. In applying these tests in the pregnant situation, it is therefore important that clinicians be aware of how pregnancy itself may alter the test results. Ultrasound promises to become established as a pivotal diagnostic test for both DVT and PE in pregnancy. The technology for compression ultrasound is available in most obstetrics units. The excellent sensitivity and specificity of compression ultrasound, combined with the modest demands on technique and interpretation, offer the potential of 24-hour access to a non-invasive and accurate objective test for occlusive proximal DVT. It should find a place in the diagnostic repertoire of all clinicians practising obstetric ultrasound.

\section{REFERENCES}

*Aitken AG \& Godden DJ (1987) Real-time ultrasound diagnosis of deep vein thrombosis: a comparison with venography. Clinical Radiology 38: 309-313.

Allbert JR, Roberts WE, Hess LW \& Morrison JC (1991) Effect of pregnancy on the accuracy of light reflection rheography. American Journal of Obstetrics and Gynecology 165: 1745-1747.

Anderson DR, Lensing AW, Wells PS et al (1993) Limitations of impedence plethysmography in the diagnosis of clinically suspected deep vein thrombosis. Annals of Internal Medicine 118: 25-30.

Bell WR, Simon TL \& DeMets DL (1977) The clinical features of submassive and massive pulmonary emboli. American Journal of Medicine 62: 355-359.

*Bergqvist D \& Hedner U (1983) Pregnancy and venous thrombo-embolism. Acta Obstetricia Gynaecologica Scandinavica 62: 449-453.

Bettman MA \& Paulin S (1977) Less phlebography: the incidence, nature and modification of undesirable side effects, Radiology 122: 101-104.

Bounameaux H, Cirafici P, De Meorloose P et al (1991) Measurement of D-Dimer in plasma as a diagnostic aid in suspected pulmonary embolism. Lancet 337: 196-200.

Cogo A, Lensing AW, Prandoni P \& Hirsh J (1993) Distribution of thrombosis in patients with symptomatic deep vein thrombosis. Implications for simplifying the diagnostic process with compression ultrasound. Archives of Internal Medicine 153: 2777-2780.

Cohen JR, Tymon R, Pillari G \& Johnson H (1988) Regional anatomical differences in the venographic occurrence of deep venous thrombosis and long-term follow-up. Journal of Cardiovascular Surgery 29: 547-551.

Comerota AJ, Katz NL \& Homayoun AH (1993) Venous duplex imaging of acute deep venous thrombosis. Haemostasis 23 (supplement): S61-S71.

Couson F, Bounameaux C \& Didier D (1993) Influence of variability of interpretation of contrast venography for screening of postoperative deep venous thrombosis on the results of a thromboprophylactic study. Thrombosis and Haemostasis 70: 573-575.

Cronan JJ, Dorfman GS, Scola FH et al (1987) Deep venous thrombosis: US assessment using vein compression. Radiology 162: 191-194.

*Cronan JJ (1993) Venous thromboembolic disease. Radiology 186: 619-630.

Demers C, Ginsberg JS, Johnston M \& Brill-Edwards P (1992) D-Dimer and thrombin-antithrombin III complexes in patients with clinically suspected pulmonary embolism. Thrombosis and Haemostasis 67: 408-412.

DHSS (Department of Health, Welsh office, Scottish Home and Health Department and Department of Health and Social Services, Northern Ireland) (1996) Confidential Enquiries into Maternal Deaths in the United Kingdom 1990-1993. London: HMSO.

Duddy MJ \& McHugo JM (1991) Duplex ultrasound of the common femoral vein in pregnancy. British Journal of Radiology 64: 785-791.

Dunnihoo DR, Gallaspy JW, Wise RB \& Otterson WN (1991) Postpartum ovarian vein thrombophlebitis: a review. Obstetric and Gynaecological Survey 46: 415-427. 
Effeney DJ, Friedman MB \& Gooding GAW (1983) Iliofemoral venous thrombosis: real-time ultrasound diagnosis, normal criteria, and clinical application. Radiology 150: 787-792.

Erdman WA, Jayson HT, Redman HC et al (1990) Deep venous thrombosis of extremities: Role of MR imaging in the diagnosis. Radiology 174: 425-431.

Evans AJ, Sostman HD, Knelson MH et al (1993) Detection of deep venous thrombosis: Prospective comparison of MR imaging with contrast venography. American Journal of Radiology 161: $131-139$.

Fairbarn JF (1963) Edema of the extremies caused primarily by regional abnormalities. Circulation 27: $981-988$.

Francalanci I, Comeglio P, Liotta AA et al (1995) D-dimer concentrations during normal pregnancy as measured by ELISA. Thrombosis Research 95: 399-405.

Genton E \& Turpie AGG (1980) Venous thromboembolism associated with gynaecologic surgery. Clinics in Obstetrics and Gynaecology 23: 209-241.

Ginsberg JS, Tumer C, Brill-Edwards P et al (1988) Pseudothrombosis in pregnancy. Canadian Medical Association Journal 139: 409-410.

* Ginsberg JS, Hirsh J, Rainbow AJ et al (1989a) Risks to the fetus of radiological procedures used in the diagnosis of maternal venous thromboembolic disease. Thrombosis and Haemostasis 61: 189-196.

Ginsberg JS, Hirsh J, Turner DC et al (1989b) Risks to the fetus of anticoagulant therapy during pregnancy. Thrombosis and Haemostasis 61: 197-203.

Greer IA, Barry J, Macklon NS \& Allan PL (1990) Diagnosis of deep venous thrombosis in pregnancy: a new role for diagnostic ultrasound. British Journal of Obstetrics and Gynaecology 97: 53-57.

*Heijboer H, Büller HR, Lensing AWA et al (1993) A comparison of real-time compression ultrasonography with impedance plethysmography for the diagnosis of deep vein thrombosis in symptomatic outpatients New England Journal of Medicine 329: 1365-1369.

Huisman MV, Buller HR, Ten Cate JW \& Vreeken J (1986) Serial impedance plethysmography for suspected deep venous thrombosis in outpatients. New England Journal of Medicine 314: 823-828.

Hull R van Acken WG, Hirsh J et al (1976) Impedence plethysmography using the occlusive cuff technique in the diagnosis of venous thrombosis. Circulation 53: 696-700.

Hull RD, Raskob GE \& LeClerc JR (1984) The diagnosis of clinically suspected venous thrombosis. Clinics in Chest Medicine 5: 439-456.

Hull RD, Hirsh J, Carter CJ et al (1985) Diagnostic efficacy of impedence plethysmography for clinical suspected deep vein thrombosis. A randomised trial. Annals of Internal Medicine 102: 21-28.

*Hull RD, Raskob GE, Ginsberg JS et al (1994) Non-invasive strategy for the treatment of patients with suspected pulmonary embolism. Archives of Internal Medicine 154: 289-297.

Illescas FF, Leclerc J, Rosenthal L et al (1993) Interobserver variability in the interpretation of contrast venography, technetium-99 $\mathrm{m}$ red blood cell venography and impedance plethysmography for deep vein thrombosis. Journal of the Canadian Association of Radiology 41: 264-269.

Juni JE \& Alavi A (1991) Lung scanning in the diagnosis of pulmonary embolism: The emperor redressed. Seminars in Nuclear Medicine 21: 281-296.

Kanal E, Shellock FG \& Talagala L (1990) Safety considerations in MR imaging. Radiology 176:593-606.

Kearon C \& Hirsh J (1995) The diagnosis of pulmonary embolism. Haemostasis 25: 72-87.

Killewich L, Bedford GR, Beach KW \& Strandness DE (1988) Diagnosis of deep vein thrombosis. A prospective study comparing duplex scanning to contrast venography. Circulation 79: 8104814 .

Lagerstedt CI, Olșson CG, Fagher BO et al (1985) Need for long-term anticoagulant treatiment in symptomatic calf-vein thrombosis. Lancet ii: 515-518.

*Lensing AWA, Prandoni P, Brandjes D et al (1989) Detection of deep vein thrombosis by real-time Bmode ultrasonography. New England Journal of Medicine 320: 342-345.

Lohr JM, Kerr TM, Lutter KS, Cranley RD et al (1991) Lower extremity calf thrombosis: to treat or not to treat? Journal of Vascular Surgery 14: 618-623.

Lohr JM, James KV, Deshmukh RM \& Hasselfeld KA (1995) Calf vein thrombi are not a benign finding. American Journal of Surgery 170: 86-90.

Machi J, Sigel B, Beitler JC et al (1983) Relation of in-vivo blood flow to ultrasound echogenicity. Journal of Clinical Ultrasound 11: 3-10. 
Macklon NS \& Greer IA (1996) Venous thromboembolic disease in obstetrics and gynaecology: the Scottish experience. Scottish Medical Journal 41: 83-86.

*Macklon NS, Greer IA \& Bowman AB (1997) An ultrasound study of gestational and postural changes in the deep venous system of the leg in pregnancy. British Journal of Obstetrics and Gynaecology 104: 191-197.

Markel A, Manzo RA, Bergelin RO \& Strandness DE (1992) Pattern and distribution of thrombi in acute venous thrombosis. Archives of Surgery 127: 305-309.

Mattos MA, Londrey GL \& Leutz DW (1992) Color-flow duplex scanning for the surveillance and diagnosis of acute deep venous thrombosis. Journal of Vascular Surgery 15: 366-376.

Meibers DJ, Baldridge ED, Ruoff BA et al (1988) The significance of calf muscle venous thrombosis. Journal of Vascular Surgery 12: 143-149.

Mills SR, Jackson DC, Older RA et al (1980) The incidence, etiologies, and avoidance of complications of pulmonary angiography in a large series. Radiology 136: 295-299.

Munsick RA \& Gillanders LA (1981) A review of the syndrome of puerperal ovarian vein thrombophlebitis. Obstetrics and Gynaecological Survey 109: 47-66.

Murphy TP \& Cronan JJ (1990) Evolution of deep venous thrombosis: a prospective evaluation with ultrasound. Radiology 177: 543-548.

Nymen U (1993) Diagnostic strategies in acute pulmonary embolism. Haemostasis 23(supplement): S220-S226.

Perlmutt LM, Braun SD, Newman GE et al (1987) Pulmonary arteriography in the high risk patient. Radiology 162: 187-189.

Pezzulo JA, Perkins AB \& Cronan JJ (1996) Symptomatic deep vein thrombosis: diagnosis with limited compression ultrasound. Radiology 198: 67-70.

Philbrick JT \& Becker DM (1988) Calf deep venous thrombosis: a wolf in sheep's clothing? Archives of Internal Medicine 148: 2131-2138.

*PIOPED Investigators (1990) Value of the ventilation/perfusion scan in acute pulmonary embolism. Results of the prospective investigation of pulmonary embolism diagnosis (PIOPED) Journal of the American Medical Association 263: 2753-2759.

Polak JF \& Wilkinson DL (1991) Ultrasonographic diagnosis of symptomatic deep venous thrombosis in pregnancy. American Journal of Obstetrics and Gynecology 165: 625-629.

Ramsey LE (1983) Impact of venography on the diagnosis and management of deep venous thrombosis: British Medical Journal 286: 698-699.

Rose SC, Zwiebel WJ \& Nelson BD (1990) Symptomatic lower extremity deep venous thrombosis: accuracy, limitations, and role of color duplex flow imaging in diagnosis. Radiology 175: 639-644.

Sarpa MS, Messina LM, Smith M et al (1989) Significance of a negative duplex scan in patients suspected of having acute deep venous thrombosis of the lower extremity. Journal of Vascular Technology 13: 222-26.

Simons GR, Piwnica-Worm DR \& Goldhaber SZ (1993) Ovarian vein thrombosis. American Heart Journal 126: 641-647.

Spritzer CE, Sostman HD, Wilkes DC \& Coleman RE (1990) Deep venous thrombosis: Experience with gradient-echo MR imaging in 66 patients. Radiology 177: 235-241.

Spritzer CE, Norconk JJ Jr, Sostman HD \& Coleman RE (1993) Detection of deep venous thrombosis by magnetic resonance imaging. Chest 104: 54-60.

Spritzer CF, Evans AC \& Kay HH (1995) Magnetic resonance imaging of deep venous thrombosis in pregnant women with lower extremity edema. Obstetrics and Gynecology 85: 603-607.

*Stein PD, Russel RD \& Pineo G (1995) Strategy that includes serial noninvasive leg tests for diagnosis of thromboembolic disease in patients with suspected acute pulmonary embolism based in data from PIOPED. Archives of Internal Medicine 155: 2101-2104.

Szucs MM, Brooks HL \& Grossman W (1971) Diagnostic sensitivity of laboratory findings in acute pulmonary embolism. Annals of Internal Medicine 74: 161-166.

Teigen CL, Maus TP, Sheedy PF et al (1993) Pulmonary embolism: diagnosis with electron beam CT. Radiology 188: 839-845.

Thomas PRS, Butler CM, Bowman J et al (1991) Light reflection rheology: an effective non-invasive technique for screening patients with suspected deep venous thrombosis. British Journal of Surgery 78: 207-209.

Vaccaro JP, Cronan JJ \& Dorfman GS (1990) Outcome analysis of patients with normal compression US examinations. Radiology 175: 645-649.

Velchik MG, Tobin M \& McCarthy K (1989) Nonthromboembolic causes of high-probability scans. American Journal of Physiology and Imaging 4: 32-38. 
Villasanta U (1965) Thromboembolic disease in pregnancy. American Journal of Obstetrics and Gynecology 93: 142-160.

Wheeler HB \& Anderson FA (1995) Diagnostic methods for deep vein thrombosis. Haemostasis 25: 6-26.

Wheeler HB, Anderson FA Jr, Cardullo PA et al (1982) Suspected deep vein thrombosis: Management by impedance plethysmography. Archives of Surgery 117: 1206-1209.

Witlin AG \& Sibai BM (1995) Postpartum ovarian vein thrombosis after vaginal delivery: a report of 11 cases. Obstetrics and Gynaecology 85: 775-780. 